5 Research Square
Preprints are preliminary reports that have not undergone peer review.

They should not be considered conclusive, used to inform clinical practice, or referenced by the media as validated information.

\title{
The impact of regional socioeconomic deprivation on late HIV diagnoses: a cross-sectional study in Germany
}

\section{Annemarie PANTKE ( $\sim$ pantkea@rki.de)}

HIV/AIDS, STI and Blood-borne Infections, Robert Koch Institute

\section{Uwe KOPPE}

HIV/AIDS, STI and Blood-borne Infections, Robert Koch Institute

\section{Matthias AN DER HEIDEN}

HIV/AIDS, STI and Blood-borne Infections, Robert Koch Institute

\section{Niels MICHALSKI}

Social Determinants of Health, Robert Koch Institute

\section{Jens HOEBEL}

Social Determinants of Health, Robert Koch Institute

\section{Viviane BREMER}

HIV/AIDS, STI and Blood-borne Infections, Robert Koch Institute

\section{Barbara GUNSENHEIMER-BARTMEYER}

HIV/AIDS, STI and Blood-borne Infections, Robert Koch Institute

\section{Norbert BANNERT}

HIV and Other Retroviruses, Robert Koch Institute

\section{Kirsten HANKE}

HIV and Other Retroviruses, Robert Koch Institute

\section{Research Article}

Keywords: late diagnosis, AIDS, socioeconomic factors, social inequalities, heterosexuals, men who have sex with men

Posted Date: November 3rd, 2021

DOl: https://doi.org/10.21203/rs.3.rs-990466/v1

License: (c) (1) This work is licensed under a Creative Commons Attribution 4.0 International License. Read Full License 


\section{Abstract}

Background: Late HIV diagnoses are associated with significantly poorer health outcomes and facilitate onward transmission. The proportion of HIV infections diagnosed at advanced stages has remained continuously high in Germany. This study examined the effect of regional socioeconomic deprivation on late HIV presentation.

Methods: We used data from the national statutory notification of newly diagnosed HIV cases collected between 2011 and 2018 with further information on the timing of diagnosis determined by the BEDCapture-ELISA test (BED-CEIA) and diagnosing physicians. Data on regional socioeconomic deprivation were derived from the German Index of Socioeconomic Deprivation (GISD). Outcome measures were a long-standing infection based on the BED-CEIA result or an infection at the stage of AIDS. The effect of socioeconomic deprivation on the timing of diagnosis was analysed using multivariable Poisson regression models with cluster-robust error variance.

Results: Overall, $66.2 \%(n=6,185)$ of the patients were diagnosed with a long-standing infection and $13.1 \%(n=1,536)$ with AIDS. The proportion of late diagnoses was higher among persons with heterosexual contact compared to men who have sex with men (MSM) (76.5\% long-standing and $16.4 \%$ AIDS vs. $62.4 \%$ long-standing and $11.9 \%$ AIDS). MSM living in highly deprived countryside regions were more likely to have a long-standing infection (aPR: 1.16, 95\% $\mathrm{Cl} 1.05-1.28$ ) as well as AIDS (aPR: 1.41, $95 \% \mathrm{Cl} 1.08-1.85)$ at the time of diagnosis compared to MSM in less deprived countryside regions. No differences were observed among MSM from urban areas, and no differences overall among persons with heterosexual contact.

Conclusions: An effect of socioeconomic deprivation on late HIV presentation was found only in MSM from countryside regions. We suggest that efforts in promoting HIV awareness and regular HIV testing are increased for heterosexual persons irrespective of socioeconomic background, and for MSM with a focus on persons living in deprived regions in the countryside.

\section{Background}

The time between an HIV infection and its diagnosis can vary widely across individuals. An undiagnosed and/or untreated infection advances with a progressive depletion of CD4 cells and a continuous decrease in immune functioning [1]. This progression can be prevented by antiretroviral therapy (ART) in most patients [2]. Late HIV diagnoses and a delayed commencement of ART therefore pose severe health risks to the individual and are also concerning on the population health level.

Persons with a deferred start of ART have a greater decline of CD4 cells and a significantly increased risk of developing AIDS-defining illnesses compared to people with an early start of ART [3]. Late presentation is also associated with substantially higher medical health care costs resulting from increased treatment needs of patients with advanced HIV infections [4,5]. On the population health level, late diagnoses are concerning as they facilitate further transmission through individuals who are unaware of their status [6]. 
Early diagnosis and treatment furthermore play a pivotal role in controlling HIV transmission in populations as effective ART leads to viral suppression and the practical elimination of a risk for onward sexual transmission [7]. In this regard, UNAIDS had set the 90-90-90 targets that were extended to the 9595-95 targets, aiming to have diagnosed $95 \%$ of all HIV positive people, provided ART for $95 \%$ of all those diagnosed and achieved viral suppression for $95 \%$ of those treated by 2030 [8].

Despite continuous efforts with respect to awareness campaigns and an increased focus on testing [9], in Europe an estimated $40-60 \%$ of persons is diagnosed with a CD 4 cell count below 350 cells $/ \mathrm{mm}^{3}$ and/or has experienced an AIDS-defining illness at the time of diagnosis [10]. The highest proportions are found in the age groups of 50 years and older and among people who acquired HIV via heterosexual contact $[10,11]$. A similar distribution can be observed in Germany [12]. In 2019, around half of the HIV infections were diagnosed at later stages with $34 \%$ at the stage of advanced immunodeficiency and $15 \%$ with AIDS. These numbers have remained continuously high since the early 2000s. Here, the highest proportions of late diagnoses were also found among the older age groups, persons with heterosexual contact as well as migrants from Sub-Saharan Africa and Asia and the Pacific $[12,13]$.

In order to reduce the number of late HIV diagnoses, it is necessary to gain a better understanding of the underlying factors that play a role in HIV health behaviours. Socioeconomic status (SES) is a factor whose impact on health has been widely investigated. Whether measured by poverty, education, social class or other indicators, low SES predicts worse health for a wide range of health behaviours and outcomes [14]. For HIV and AIDS, some studies underline this link, showing that individuals of lower SES are at a disproportionately higher risk for contracting HIV, presenting at later stages and also have higher mortality rates [15-17]. In Germany, this relationship has not been examined yet. As the HIV surveillance system does not include information on socioeconomic factors at the individual patient level, this study utilised the German Index of Socioeconomic Deprivation (GISD), which describes socioeconomic inequalities at different regional levels in Germany [18]. The GISD was generated at the levels of associations of municipalities, administrative districts and administrative regions and is based on the sub-dimensions of education, occupation, and income. The calculated deprivation scores depict the socio-spatial burden in the respective area and can be utilised to investigate the link between regional socioeconomic deprivation and health.

Using the GISD, this study aimed to examine whether socioeconomic deprivation in Germany affects the timing of HIV diagnosis. Knowledge about its potential impact on late HIV presentation can be used to improve and render public health strategies aimed at promoting HIV awareness and early testing behaviours more effectively.

\section{Methods}

\section{Study design}


This study is a cross-sectional study using data from the "InzSurv-HIV" study at the Robert Koch Institute (RKI) in Germany. The dataset contains pseudonymised information from the national statutory notification of newly diagnosed HIV cases that are directly reported to the RKI. For about $60 \%$ of the new diagnoses, the RKI additionally receives dried serum or plasma spots from participating laboratories which are then tested for recency of infection using the BED-Capture-ELISA (BED-CEIA) [19, 20]. Cases are classified as recent or long-standing with a cut-off point that corresponds to a mean duration of approximately five months after the infection [21]. Since the BED-CEIA might produce false-recent results in people with advanced immunodeficiency, cases classified as recent while diagnosed with an AIDSdefining condition were considered long-standing infections. Included in this analysis were all cases from 2011 to 2018. Excluded were cases with the age below 15 years at the time of diagnosis as well as cases with a transmission mode of blood transfusion, haemophilia, occupational exposition and mother to child transmission due to sparse data. The GISD data were linked to this dataset on the basis of the 3digit ZIP code with each patient receiving the respective socioeconomic deprivation score of their residential area. In addition to that, data on city population size were derived from the Federal Statistical Office of Germany and merged to the dataset [22].

\section{Outcomes and covariates}

The risk for late HIV diagnoses was examined at two time points: The first outcome was an HIV diagnosis at the stage of a long-standing infection as determined by the BED-CEIA assay. A long-standing HIV infection is defined as an infection that has been diagnosed after a mean duration of more than approximately 20 weeks ( $=5$ months) since it was acquired [21]. The second outcome was an HIV infection that was diagnosed at the clinical stage of AIDS. This outcome was derived from the CDC classification system for HIV diagnoses in which category $\mathrm{C}$ classifies patients who have already developed an AIDS-defining condition at the time of their HIV diagnosis [23]. The classification was done by the diagnosing physician and reported to the RKI via the statutory notification.

While the first outcome identifies all cases diagnosed after the acute phase, the second outcome is more specific and only looks at diagnoses received at a further advanced stage with an AIDS-defining condition. The two outcome measures depict different points in time with regard to HIV progression and were analysed separately.

The exposure of interest was regional socioeconomic deprivation using the GISD score, which is based on the sub-dimensions of education, occupation, and income $[18,24]$. The calculated index values were divided into quintiles and then grouped into three categories, representing low deprivation (lowest quintile), medium deprivation (middle three quintiles) and high deprivation (highest quintile). High socioeconomic deprivation can be equally understood as a lack of socioeconomic resources or low socioeconomic status [18].

Further variables included in the analysis were transmission mode (men who have sex with men (MSM), persons with heterosexual contact (HET), persons who inject drugs (PWID)), sex (male, female), approximated age at the time of infection (15-19, 20-29, 30-39, 40-49, 50-59, 60-69, >69 years), region of 
origin (Western and Central Europe, Asia and the Pacific, Caribbean, Eastern Europe and Central Asia, Latin America, Middle East and North Africa, North America, Sub-Saharan Africa) and city size (countryside: $<100 \mathrm{k}$ residents, town: $100 \mathrm{k}-<1$ million residents, major city: $>=1$ million residents). As the age at the time of infection is unknown, this variable was approximated from the available age at the time of diagnosis by subtracting the approximate median progression periods on the basis of the CDC classification system [23]. We subtracted one age year from cases classified as category A (acute, asymptomatic infection), five age years from cases classified as category $B$ (chronic, symptomatic infection), and ten age years from cases classified as category $C$ (AIDS) $[23,25,26]$. No age years were subtracted from cases younger than 30 years at the time of diagnosis to avoid computing unrealistic outliers in the lower end age groups. Even though the median time periods until the development of AIDS decrease with older age [25], this procedure is justified as persons who are diagnosed with an AIDSdefining illness at a younger age usually have shorter lags between HIV contraction and diagnosis, while persons diagnosed with AIDS in the older age groups tend to have longer lags.

\section{Statistical analysis}

Separate complete case analyses were conducted for both outcome measures. To estimate the effect of socioeconomic deprivation on late presentation, prevalence ratios using multivariable Poisson regressions with ZIP code cluster-robust error variance to account for the individual and regional level data structure were computed. The models were adjusted for the variables of transmission mode, sex, approximated age at the time of infection, region of origin and city size. These variables were identified as confounders by a directed acyclic graph, which was created on the basis of prior research (see Figure S1, Additional file 1). Stratified analyses were performed for MSM and persons with heterosexual contact as effect modification was expected for these groups due to their differing health seeking behaviours with respect to HIV [13]. In the case of discernible effects, an interaction term between the GISD and city size was planned to be included in the models to examine a potential interaction of these variables, seeing that HIV support structures in urban areas differ from those in rural areas [27]. Adjusted prevalence ratios $(\mathrm{aPR})$ and $95 \%$ confidence intervals $(95 \% \mathrm{Cl})$ were calculated.

\section{Results}

After excluding all cases with missing data, a total of 9,342 cases with BED-CEIA results and 11,774 cases with a documented CDC category were included in the analysis. Among the 9,342 patients with available BED-CEIA test results, $66.2 \%$ had an HIV infection classified as long-standing at the time of diagnosis (Table 1). Patients in the highest socioeconomic deprivation quintile showed a slightly higher proportion of long-standing infections (68.9\%) compared to the low $(66.2 \%)$ and medium deprivation quintiles (65.8\%). With regard to the other variables included in the analysis, the highest proportions of long-standing infections were found in the heterosexual transmission group $(76.5 \%)$ and among migrants from Sub-Saharan Africa (79.2\%). Among the 11,774 patients with a documented CDC category, 13.1\% had reached the clinical stage of AIDS at the time of their diagnosis (Table 1). Patients living in regions with the highest socioeconomic deprivation were more often diagnosed with an AIDS-defining condition 
(16.2\%) than patients of low (12.7\%) and medium deprivation (12.7\%) background. Concerning the other variables, the highest proportions of AIDS-defining conditions were observed in the heterosexual transmission group (16.4\%) and patients between 60 and 69 years at the time of diagnosis $(28.2 \%)$ (Table 1). 
Table 1 Baseline data of newly diagnosed HIV cases by BED-CEIA ${ }^{1}$ recency test and CDC category

$N=11,774$

$\mathrm{N}=9,342$

\begin{tabular}{llll}
$\begin{array}{l}\text { Long- } \\
\text { standing } \\
\text { infection }\end{array}$ & $\begin{array}{l}\text { Recent } \\
\text { infection }\end{array}$ & AIDS & No AIDS \\
\hline $6,185(66.2 \%)$ & $3,157(33.8 \%)$ & $\begin{array}{l}1,536 \\
(13.1 \%)\end{array}$ & $\begin{array}{l}10,238 \\
(86.9 \%)\end{array}$
\end{tabular}

$\mathrm{GISD}^{2}$

Low deprivation

$\begin{array}{cc}1,663(66.2 \%) & 849 \\ (33.8 \%)\end{array}$

410

$(12.7 \%)$

2,812

(33.8\%)

Medium deprivation

$3,909(65.8 \%) \quad 2,031$ (34.2\%)

929

(12.7\%)

(87.3\%)

High deprivation

$\begin{array}{lc}613 & 277 \\ (68.9 \%) & (31.1 \%)\end{array}$

197

(16.2\%)

6,404

(87.3\%)

613
$(68.9 \%)$

$(31.1 \%)$

(16.2\%)

1,022

(83.8\%)

Transmission mode

$\mathrm{MSM}^{3}$

$4,061(62.4 \%) \quad 2,450(37.6 \%)$

973

(11.9\%)

7,225

$\mathrm{HET}^{4}$

$1,905(76.5 \%)$

586

(23.5\%)

509

(16.4\%)

(88.1\%)

PWID $^{5}$

219

(64.4\%)

121

(35.6\%)

54

$(11.7 \%)$

2,604

(83.6\%)

Sex

Male

$4,850(64.2 \%)$

$2,708(35.8 \%)$

1,215

8,348

(12.7\%)

$(87.3 \%)$

Female

$\begin{array}{cc}1,335(74.8 \%) & 449 \\ & (25.2 \%)\end{array}$

321
$(14.5 \%)$

1,890

(85.5\%)

Age (time of diagnosis)

15 to 19

20 to 29

30 to 39

40 to 49
92

(51.7\%)

$1,512(60.2 \%) \quad 1,000$ (39.8\%)

86

(48.3\%)

$8(4.4 \%)$

176

(95.6\%)

153

(5.1\%)

2,850

(94.9\%)

3,390

(88.8\%)

(11.2\%)

2,366

490
$(17.2 \%)$

(82.8\%) 


\begin{tabular}{|c|c|c|c|c|}
\hline 50 to 59 & $\begin{array}{c}746 \\
(71.3 \%)\end{array}$ & $\begin{array}{c}301 \\
(28.7 \%)\end{array}$ & $\begin{array}{c}317 \\
(22.5 \%)\end{array}$ & $\begin{array}{l}1,090 \\
(77.5 \%)\end{array}$ \\
\hline 60 to 69 & $\begin{array}{c}211 \\
(73.3 \%)\end{array}$ & $\begin{array}{c}77 \\
(26.7 \%)\end{array}$ & $\begin{array}{c}113 \\
(28.2 \%)\end{array}$ & $\begin{array}{c}288 \\
(71.8 \%)\end{array}$ \\
\hline$>69$ & $\begin{array}{c}56 \\
(66.7 \%)\end{array}$ & $\begin{array}{c}28 \\
(33.3 \%)\end{array}$ & $\begin{array}{c}28 \\
(26.4 \%)\end{array}$ & $\begin{array}{c}78 \\
(73.6 \%)\end{array}$ \\
\hline \multicolumn{5}{|l|}{ Approx. age (time of infection) } \\
\hline 15 to 19 & $\begin{array}{c}92 \\
(51.7 \%)\end{array}$ & $\begin{array}{c}86 \\
(48.3 \%)\end{array}$ & $8 \quad(4.4 \%)$ & $\begin{array}{c}176 \\
(95.6 \%)\end{array}$ \\
\hline 20 to 29 & $2,267(66.5 \%)$ & $1,143(33.5 \%)$ & $\begin{array}{c}580 \\
(14.2 \%)\end{array}$ & $\begin{array}{l}3,510 \\
(85.8 \%)\end{array}$ \\
\hline 30 to 39 & $1,957(66.6 \%)$ & $\begin{array}{c}980 \\
(33.4 \%)\end{array}$ & $\begin{array}{c}490 \\
(13.1 \%)\end{array}$ & $\begin{array}{l}3,245 \\
(86.9 \%)\end{array}$ \\
\hline 40 to 49 & $1,278(67.5 \%)$ & $\begin{array}{c}615 \\
(32.5 \%)\end{array}$ & $\begin{array}{c}317 \\
(12.6 \%)\end{array}$ & $\begin{array}{l}2,209 \\
(87.4 \%)\end{array}$ \\
\hline 50 to 59 & $\begin{array}{c}446 \\
(64.3 \%)\end{array}$ & $\begin{array}{c}248 \\
(35.7 \%)\end{array}$ & $\begin{array}{c}113 \\
(12.0 \%)\end{array}$ & $\begin{array}{c}831 \\
(88.0 \%)\end{array}$ \\
\hline 60 to 69 & $\begin{array}{c}119 \\
(65.0 \%)\end{array}$ & $\begin{array}{c}64 \\
(35.0 \%)\end{array}$ & $\begin{array}{c}27 \\
(11.2 \%)\end{array}$ & $\begin{array}{c}214 \\
(88.8 \%)\end{array}$ \\
\hline$>69$ & $\begin{array}{c}26 \\
(55.3 \%)\end{array}$ & $\begin{array}{c}21 \\
(44.7 \%)\end{array}$ & $\begin{array}{c}1 \\
(1.9 \%)\end{array}$ & $\begin{array}{c}53 \\
(98.1 \%)\end{array}$ \\
\hline \multicolumn{5}{|l|}{ Region of origin } \\
\hline Western and Central Europe & $4,462(63.2 \%)$ & $2,600(36.8 \%)$ & $\begin{array}{l}1,152 \\
(12.8 \%)\end{array}$ & $\begin{array}{l}7,859 \\
(87.2 \%)\end{array}$ \\
\hline Asia and the Pacific & $\begin{array}{c}195 \\
(72.5 \%)\end{array}$ & $\begin{array}{c}74 \\
(27.5 \%)\end{array}$ & $\begin{array}{c}66 \\
(19.5 \%)\end{array}$ & $\begin{array}{l}272 \\
(80.5 \%)\end{array}$ \\
\hline Caribbean & $\begin{array}{c}25 \\
(69.4 \%)\end{array}$ & $\begin{array}{c}11 \\
(30.6 \%)\end{array}$ & $\begin{array}{c}4 \\
(8.7 \%)\end{array}$ & $\begin{array}{c}42 \\
(91.3 \%)\end{array}$ \\
\hline $\begin{array}{l}\text { Eastern Europe and Central } \\
\text { Asia }\end{array}$ & $\begin{array}{c}214 \\
(72.1 \%)\end{array}$ & $\begin{array}{c}83 \\
(27.9 \%)\end{array}$ & $\begin{array}{c}50 \\
(13.3 \%)\end{array}$ & $\begin{array}{c}327 \\
(86.7 \%)\end{array}$ \\
\hline Latin America & $\begin{array}{c}119 \\
(66.5 \%)\end{array}$ & $\begin{array}{c}60 \\
(33.5 \%)\end{array}$ & $\begin{array}{c}20 \\
(8.4 \%)\end{array}$ & $\begin{array}{l}219 \\
(91.6 \%)\end{array}$ \\
\hline $\begin{array}{l}\text { Middle East and North } \\
\text { Africa }\end{array}$ & $\begin{array}{c}107 \\
(69.9 \%)\end{array}$ & $\begin{array}{c}46 \\
(30.1 \%)\end{array}$ & $\begin{array}{c}16 \\
(8.5 \%)\end{array}$ & $\begin{array}{c}172 \\
(91.5 \%)\end{array}$ \\
\hline North America & $\begin{array}{c}25 \\
(71.4 \%)\end{array}$ & $\begin{array}{c}10 \\
(28.6 \%)\end{array}$ & $\begin{array}{c}5 \\
(11.1 \%)\end{array}$ & $\begin{array}{c}40 \\
(88.9 \%)\end{array}$ \\
\hline Sub-Saharan Africa & $\begin{array}{c}1,038 \\
(79.2 \%)\end{array}$ & $\begin{array}{c}273 \\
(20.8 \%)\end{array}$ & $\begin{array}{c}223 \\
(14.6 \%)\end{array}$ & $\begin{array}{l}1,307 \\
(85.4 \%)\end{array}$ \\
\hline
\end{tabular}


City size

\begin{tabular}{lccccc} 
Countryside <100k res. & $2,651(69.1 \%)$ & $1,184(30.9 \%)$ & $\begin{array}{c}704 \\
(14.7 \%)\end{array}$ & $\begin{array}{c}4,077 \\
(85.3 \%)\end{array}$ \\
\hline Town 100k $-<1$ million res. & $1,946(64.5 \%)$ & $1,071(35.5 \%)$ & $\begin{array}{c}456 \\
(12.5 \%)\end{array}$ & $\begin{array}{c}3,189 \\
(87.5 \%)\end{array}$ \\
\hline Major city >= 1 million res. & $1,588(63.8 \%)$ & $\begin{array}{c}902 \\
(36.2 \%)\end{array}$ & $\begin{array}{c}376 \\
(11.2 \%)\end{array}$ & $\begin{array}{c}2,972 \\
(88.8 \%)\end{array}$
\end{tabular}

${ }^{1}$ BED-CEIA BED-Capture-ELISA recency test, ${ }^{2}$ GISD German Index of Socioeconomic Deprivation,

${ }^{3} \mathrm{MSM}$ Men who have sex with men, ${ }^{4} \mathrm{HET}$ Persons with heterosexual contact, ${ }^{5} \mathrm{PWID}$ Persons who inject drugs

The results of the stratified multivariable analyses of the BED-CEIA outcome measure showed that MSM living in regions that belong to the highest socioeconomic deprivation quintile were somewhat more likely to have a long-standing infection at the time of diagnosis (aPR: $1.06,95 \% \mathrm{Cl} 0.99-1.13$ ) compared to MSM in regions of lower deprivation (Table 2). In the heterosexual transmission group, no differences between persons of high, medium or low socioeconomic deprivation background were observed (Table 2). An interaction term between the GISD and city size was included in the regression model of the MSM stratum, which showed that these variables interact with each other. While MSM from highly and medium deprived countryside areas were more likely to have a long-standing HIV infection at the time of diagnosis (aPR: 1.16, 95\% Cl 1.05-1.28; aPR: 1.09, 95\% Cl 1.01-1.18) compared to MSM from countryside areas of low socioeconomic deprivation (Table 3), no differences in the adjusted prevalence ratios were observed between MSM from towns or major cities with different deprivation levels (Table 3).

The results of the stratified multivariable analyses of the CDC outcome measure showed that MSM living in regions belonging to the highest socioeconomic deprivation quintile were somewhat more likely to have reached the clinical stage of AIDS at the time of diagnosis (aPR: 1.21, 95\% Cl 0.98-1.50) compared to MSM in regions of lower deprivation (Table 4). In the heterosexual transmission group, again no differences between persons of high, medium or low socioeconomic deprivation background were observed (Table 4). In this analysis, an interaction term between the GISD and city size was also included in the regression model of the MSM stratum, which again revealed an interaction of the same type between these variables. While MSM living in highly deprived countryside areas were more likely to have 
Table 2 Multivariable analysis of long-standing HIV infections stratified for transmission mode

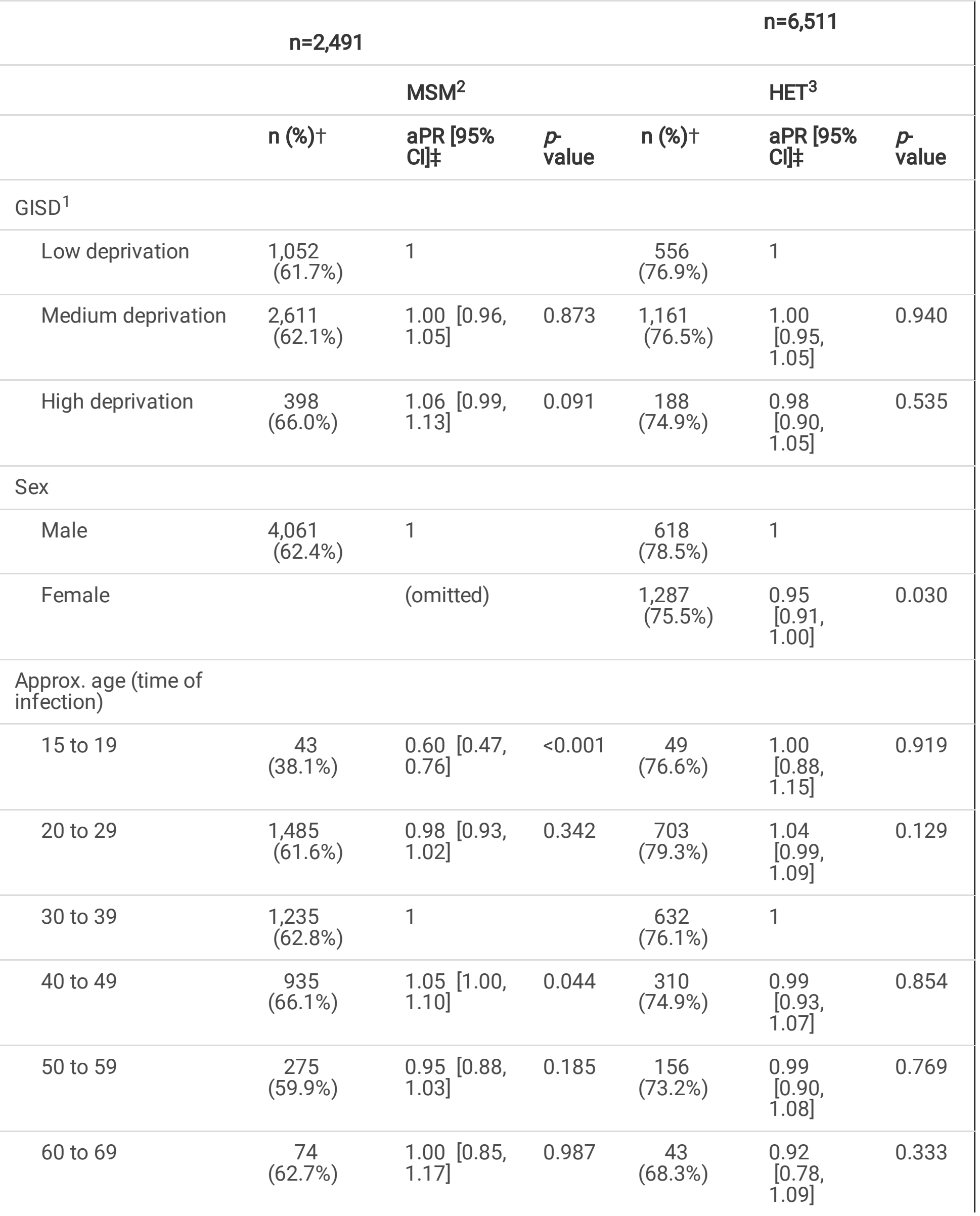


$>69$

\begin{tabular}{|c|c|c|c|c|}
\hline $\begin{array}{c}14 \\
(50.0 \%)\end{array}$ & $\begin{array}{l}0.80[0.58, \\
1.11]\end{array}$ & 0.181 & $\begin{array}{c}12 \\
(63.2 \%)\end{array}$ & $\begin{array}{l}0.86 \\
{[0.60,} \\
1.24]\end{array}$ \\
\hline
\end{tabular}

Region of origin

\begin{tabular}{|c|c|c|c|c|c|c|}
\hline $\begin{array}{l}\text { Western and Central } \\
\text { Europe }\end{array}$ & $\begin{array}{l}3,685 \\
(62.2 \%)\end{array}$ & 1 & & $\begin{array}{c}631 \\
(70.8 \%)\end{array}$ & 1 & \\
\hline Asia and the Pacific & $\begin{array}{c}103 \\
(65.6 \%)\end{array}$ & $\begin{array}{l}1.07[0.96, \\
1.20]\end{array}$ & 0.222 & $\begin{array}{c}89 \\
(81.7 \%)\end{array}$ & $\begin{array}{l}1.16 \\
{[1.04} \\
1.28]\end{array}$ & 0.007 \\
\hline Caribbean & $\begin{array}{c}14 \\
(70.0 \%)\end{array}$ & $\begin{array}{l}1.14[0.84, \\
1.54]\end{array}$ & 0.407 & $\begin{array}{c}11 \\
(68.8 \%)\end{array}$ & $\begin{array}{l}0.98 \\
{[0.70,} \\
1.37]\end{array}$ & 0.889 \\
\hline $\begin{array}{l}\text { Eastern Europe and } \\
\text { Central Asia }\end{array}$ & $\begin{array}{c}44 \\
(57.1 \%)\end{array}$ & $\begin{array}{l}0.93[0.77, \\
1.12]\end{array}$ & 0.433 & $\begin{array}{c}109 \\
(77.3 \%)\end{array}$ & $\begin{array}{l}1.08 \\
{[0.98} \\
1.20]\end{array}$ & 0.107 \\
\hline Latin America & $\begin{array}{c}105 \\
(66.0 \%)\end{array}$ & $\begin{array}{l}1.09[0.98, \\
1.20]\end{array}$ & 0.101 & $\begin{array}{c}13 \\
(68.4 \%)\end{array}$ & $\begin{array}{l}0.97 \\
{[0.71} \\
1.32]\end{array}$ & 0.838 \\
\hline $\begin{array}{l}\text { Middle East and } \\
\text { North Africa }\end{array}$ & $\begin{array}{c}49 \\
(62.8 \%)\end{array}$ & $\begin{array}{l}1.02[0.85, \\
1.23]\end{array}$ & 0.802 & $\begin{array}{c}54 \\
(80.6 \%)\end{array}$ & $\begin{array}{l}1.11 \\
{[0.98} \\
1.25]\end{array}$ & 0.089 \\
\hline North America & $\begin{array}{c}24 \\
(72.7 \%)\end{array}$ & $\begin{array}{l}1.19[0.92, \\
1.54]\end{array}$ & 0.191 & $\begin{array}{c}1 \\
(50.0 \%)\end{array}$ & $\begin{array}{l}0.69 \\
{[0.17} \\
2.90]\end{array}$ & 0.616 \\
\hline Sub-Saharan Africa & $\begin{array}{c}37 \\
(60.7 \%)\end{array}$ & $\begin{array}{l}1.01[0.82, \\
1.25]\end{array}$ & 0.911 & $\begin{array}{c}997 \\
(80.0 \%)\end{array}$ & $\begin{array}{l}1.12 \\
{[1.06,} \\
1.18]\end{array}$ & $<0.001$ \\
\hline
\end{tabular}

City size

\begin{tabular}{|c|c|c|c|c|c|c|}
\hline $\begin{array}{l}\text { Countryside }<100 \mathrm{k} \\
\text { res. }\end{array}$ & $\begin{array}{l}1,578 \\
(64.5 \%)\end{array}$ & $\begin{array}{l}1.06[1.00, \\
1.12]\end{array}$ & 0.041 & $\begin{array}{c}971 \\
(77.7 \%)\end{array}$ & $\begin{array}{l}1.03 \\
{[0.96,} \\
1.11]\end{array}$ & 0.397 \\
\hline $\begin{array}{l}\text { Town } 100 \mathrm{k}-<1 \\
\text { million res. }\end{array}$ & $\begin{array}{l}1,289 \\
(61.0 \%)\end{array}$ & $\begin{array}{l}1.00[0.95, \\
1.06]\end{array}$ & 0.988 & $\begin{array}{c}578 \\
(75.1 \%)\end{array}$ & $\begin{array}{l}1.00 \\
{[0.92,} \\
1.08]\end{array}$ & 0.980 \\
\hline $\begin{array}{l}\text { Major city >= } 1 \\
\text { million res. }\end{array}$ & $\begin{array}{l}1,194 \\
(61.1 \%)\end{array}$ & 1 & & $\begin{array}{c}356 \\
(75.6 \%)\end{array}$ & 1 & \\
\hline
\end{tabular}

${ }^{1}$ GISD German Index of Socioeconomic Deprivation, ${ }^{2}$ MSM Men who have sex with men,

${ }^{3} \mathrm{HET}$ Persons with heterosexual contact

TStrata specific number and proportion of long-standing infections at the time of diagnosis.

¥Prevalence ratios with corresponding $95 \%$ confidence intervals of long-standing infections at the time of diagnosis were calculated for the exposure variable GISD using stratified multivariable Poisson regression with ZIP code cluster-robust error variance (standard errors were adjusted for 626 
clusters in the MSM stratum and 562 clusters in the HET stratum). The models were adjusted for the variables of sex, approximated age at the time of infection, region of origin and city size.

\section{Table 3 Multivariable analysis of long-standing HIV infections including interaction term between GISD $^{1}$ and city size (only MSM ${ }^{2}$ )}

\section{$n=6,511$}

Countryside ( $<100 \mathrm{k}$ residents)

n (\%)†
aPR $[95 \% \mathrm{Cl}] \ddagger$

p-value $\mathrm{n}(\%)+$
Town/Major city ( $>=100 \mathrm{k}$ residents)

aPR $[95 \% \mathrm{Cl}] \ddagger \quad p$-value

\begin{tabular}{|c|c|c|c|c|c|c|c|}
\hline 234 & (59.9\%) & 1 & & 815 & $(62.2 \%)$ & 1 & \\
\hline 1,090 & (64.7\%) & $1.09[1.01,1.18]$ & 0.033 & 1,521 & $(60.4 \%)$ & $0.97[0.92,1.03]$ & 0.350 \\
\hline 251 & $(68.8 \%)$ & $1.16[1.05,1.28]$ & 0.004 & 147 & $(61.8 \%)$ & $1.00[0.92,1.09]$ & 0.953 \\
\hline
\end{tabular}

${ }^{1}$ GISD German Index of Socioeconomic Deprivation, ${ }^{2}$ MSM Men who have sex with men

TStrata specific number and proportion of long-standing infections at the time of diagnosis.

¥Prevalence ratios with corresponding $95 \%$ confidence intervals of long-standing infections at the time of diagnosis were calculated for the exposure variable GISD using multivariable Poisson regression with ZIP code cluster-robust error variance (standard errors were adjusted for 626 clusters). The model was stratified for MSM and adjusted for the variables of approximated age at the time of infection, region of origin and city size. For simplicity purposes, only strata specific effect estimates of the GISD conditional on countryside vs. town/major city are depicted. The effect estimates of the remaining covariates are nearly identical as presented in Table 2 in the MSM stratum.

developed AIDS at the time of their diagnosis (aPR: 1.41, 95\% $\mathrm{Cl} 1.08-1.85$ ) compared to MSM in less deprived countryside areas (Table 5), again no differences were observed among MSM from towns or major cities with different deprivation levels (Table 5).

\section{References}

1. Song A, Li Z, Luo Z, Lu X, Wang R, Liu L, et al. Effects of Early and Delayed Antiretroviral Therapy on 
Table 4 Multivariable analysis of infections at the stage of AIDS stratified for transmission mode

\begin{tabular}{|c|c|c|c|c|c|c|}
\hline \multicolumn{4}{|c|}{$n=3,113$} & \multicolumn{3}{|c|}{$n=8,198$} \\
\hline & \multicolumn{3}{|c|}{ MSM $^{2}$} & \multicolumn{3}{|c|}{$\mathrm{HET}^{3}$} \\
\hline & $n(\%) \dagger$ & $\begin{array}{l}\text { aPR [95\% } \\
\text { Cl] } \neq\end{array}$ & $\begin{array}{l}p \\
\text { value }\end{array}$ & $n(\%) \dagger$ & $\begin{array}{l}\text { aPR }[95 \% \\
\text { Cl] } \neq\end{array}$ & $\begin{array}{l}p- \\
\text { value }\end{array}$ \\
\hline \multicolumn{7}{|l|}{ GISD $^{1}$} \\
\hline Low deprivation & $\begin{array}{c}253 \\
(11.4 \%)\end{array}$ & 1 & & $\begin{array}{c}149 \\
(16.5 \%)\end{array}$ & 1 & \\
\hline Medium deprivation & $\begin{array}{c}594 \\
(11.5 \%)\end{array}$ & $\begin{array}{l}0.98[0.85, \\
1.13]\end{array}$ & 0.750 & $\begin{array}{c}299 \\
(16.2 \%)\end{array}$ & $\begin{array}{l}0.99 \\
{[0.82} \\
1.19]\end{array}$ & 0.923 \\
\hline High deprivation & $\begin{array}{c}126 \\
(15.6 \%)\end{array}$ & $\begin{array}{l}1.21[0.98, \\
1.50]\end{array}$ & 0.076 & $\begin{array}{c}61 \\
(16.8 \%)\end{array}$ & $\begin{array}{l}1.05 \\
{[0.79} \\
1.40]\end{array}$ & 0.735 \\
\hline \multicolumn{7}{|l|}{ Sex } \\
\hline Male & $\begin{array}{c}973 \\
(11.9 \%)\end{array}$ & 1 & & $\begin{array}{c}195 \\
(19.2 \%)\end{array}$ & 1 & \\
\hline Female & & (omitted) & & $\begin{array}{c}314 \\
(15.0 \%)\end{array}$ & $\begin{array}{l}0.72 \\
{[0.60,} \\
0.86]\end{array}$ & $<0.001$ \\
\hline
\end{tabular}

Approx. age (time of infection)

\begin{tabular}{|c|c|c|c|c|c|c|}
\hline 15 to 19 & $\begin{array}{c}4 \\
(3.5 \%)\end{array}$ & $\begin{array}{l}0.26[0.10, \\
0.89]\end{array}$ & 0.007 & $\begin{array}{c}4 \\
(5.8 \%)\end{array}$ & $\begin{array}{l}0.40 \\
{[0.16,} \\
1.02]\end{array}$ & 0.056 \\
\hline 20 to 29 & $\begin{array}{c}350 \\
(12.1 \%)\end{array}$ & $\begin{array}{l}0.94 \text { [0.82, } \\
1.07]\end{array}$ & 0.369 & $\begin{array}{c}201 \\
(19.0 \%)\end{array}$ & $\begin{array}{l}1.31 \\
{[1.07} \\
1.60]\end{array}$ & 0.008 \\
\hline 30 to 39 & $\begin{array}{c}320 \\
(12.8 \%)\end{array}$ & 1 & & $\begin{array}{c}155 \\
(15.0 \%)\end{array}$ & 1 & \\
\hline 40 to 49 & $\begin{array}{c}218 \\
(11.6 \%)\end{array}$ & $\begin{array}{l}0.89 \text { [0.76, } \\
1.05]\end{array}$ & 0.185 & $\begin{array}{c}90 \\
(16.3 \%)\end{array}$ & $\begin{array}{l}1.00 \\
{[0.78,} \\
1.27]\end{array}$ & 0.975 \\
\hline 50 to 59 & $\begin{array}{c}66 \\
(10.5 \%)\end{array}$ & $\begin{array}{l}0.80 \text { [0.62, } \\
1.03]\end{array}$ & 0.082 & $\begin{array}{c}46 \\
(15.9 \%)\end{array}$ & $\begin{array}{l}0.95 \\
{[0.68,} \\
1.33]\end{array}$ & 0.761 \\
\hline 60 to 69 & $\begin{array}{c}15 \\
(9.8 \%)\end{array}$ & $\begin{array}{l}0.74 \text { [0.45, } \\
1.22]\end{array}$ & 0.243 & $\begin{array}{c}12 \\
(13.4 \%)\end{array}$ & $\begin{array}{l}0.78 \\
{[0.45,} \\
1.35]\end{array}$ & 0.371 \\
\hline
\end{tabular}


$>69$
0
$(0.0 \%)$
(omitted)
1
$(4.6 \%)$
0.25
[0.04,
1.75]

Region of origin

\begin{tabular}{|c|c|c|c|c|c|c|}
\hline $\begin{array}{l}\text { Western and Central } \\
\text { Europe }\end{array}$ & $\begin{array}{c}896 \\
(12.0 \%)\end{array}$ & 1 & & $\begin{array}{c}215 \\
(17.5 \%)\end{array}$ & 1 & \\
\hline Asia and the Pacific & $\begin{array}{c}30 \\
(14.9 \%)\end{array}$ & $\begin{array}{l}1.26[0.90, \\
1.76]\end{array}$ & 0.173 & $\begin{array}{c}35 \\
(26.3 \%)\end{array}$ & $\begin{array}{l}1.57 \\
{[1.16,} \\
2.12]\end{array}$ & 0.003 \\
\hline Caribbean & $\begin{array}{c}1 \\
(3.7 \%)\end{array}$ & $\begin{array}{l}0.31[0.05, \\
2.15]\end{array}$ & 0.238 & $\begin{array}{c}3 \\
(15.8 \%)\end{array}$ & $\begin{array}{l}0.90 \\
{[0.33,} \\
2.49]\end{array}$ & 0.846 \\
\hline $\begin{array}{l}\text { Eastern Europe and } \\
\text { Central Asia }\end{array}$ & $\begin{array}{c}13 \\
(12.6 \%)\end{array}$ & $\begin{array}{l}1.05[0.64, \\
1.71]\end{array}$ & 0.849 & $\begin{array}{c}26 \\
(15.3 \%)\end{array}$ & $\begin{array}{l}0.90 \\
{[0.62,} \\
1.31]\end{array}$ & 0.596 \\
\hline Latin America & $\begin{array}{c}15 \\
(7.0 \%)\end{array}$ & $\begin{array}{l}0.62[0.38, \\
1.02]\end{array}$ & 0.059 & $\begin{array}{c}5 \\
(19.2 \%)\end{array}$ & $\begin{array}{l}1.03 \\
{[0.47} \\
2.29]\end{array}$ & 0.935 \\
\hline $\begin{array}{l}\text { Middle East and } \\
\text { North Africa }\end{array}$ & $\begin{array}{c}5 \\
(5.3 \%)\end{array}$ & $\begin{array}{l}0.44[0.19, \\
1.02]\end{array}$ & 0.057 & $\begin{array}{c}11 \\
(14.1 \%)\end{array}$ & $\begin{array}{l}0.71 \\
{[0.38,} \\
1.32]\end{array}$ & 0.278 \\
\hline North America & $\begin{array}{c}4 \\
(9.8 \%)\end{array}$ & $\begin{array}{l}0.88[0.36, \\
2.17]\end{array}$ & 0.789 & $\begin{array}{c}1 \\
(25.0 \%)\end{array}$ & $\begin{array}{l}1.45 \\
{[0.23,} \\
9.01]\end{array}$ & 0.692 \\
\hline Sub-Saharan Africa & $\begin{array}{c}9 \\
(12.9 \%)\end{array}$ & $\begin{array}{l}1.08[0.58, \\
2.01]\end{array}$ & 0.798 & $\begin{array}{c}213 \\
(14.7 \%)\end{array}$ & $\begin{array}{l}0.79 \\
{[0.66,} \\
0.95]\end{array}$ & 0.014 \\
\hline
\end{tabular}

City size

Countryside $<100 k$ res.

Town 100k - <1

million res.

Major city $>=1$

million res.
426

(14.1\%)

$1.37[1.16, \quad<0.001$

1.64]

253

(16.1\%)

0.90

[0.73,

1.11]

287

(11.4\%)

$1.13[0.94, \quad 0.192$

$1.35]$

150
$(16.2 \%)$

$(16.2 \%)$

0.94

[0.75,

1.17]

106
$(17.4 \%)$

1

${ }^{1}$ GISD German Index of Socioeconomic Deprivation, ${ }^{2}$ MSM Men who have sex with men,

${ }^{3} H E T$ Persons with heterosexual contact

TStrata specific number and proportion of infections at the stage of AIDS at the time of diagnosis.

¥Prevalence ratios with corresponding $95 \%$ confidence intervals of infections at the stage of AIDS at the time of diagnosis were calculated for the exposure variable GISD using stratified multivariable Poisson regression with ZIP code cluster-robust error variance (standard errors were adjusted for 644 
clusters in the MSM stratum and 592 clusters in the HET stratum). The models were adjusted for the variables of sex, approximated age at the time of infection, region of origin and city size.

Table 5 Multivariable analysis of infections at the stage of AIDS including interaction term between GISD $^{1}$ and city size (only $\mathrm{MSM}^{2}$ )

$\mathrm{n}=\mathbf{8 , 1 9 8}$

Countryside (<100k residents)

n (\%)†

Medium

deprivation
aPR $[95 \%$

$\mathrm{Cl}] \ddagger$
66

$(12.3 \%)$

1

(1)

$1.12[0.88$

1.42]

$(13.7 \%)$

92
$(17.2 \%)$

268

$1.85]$
GISD $^{1}$
High deprivation

Low deprivation
Town/Major city ( $>=100 \mathrm{k}$ residents)

n (\%)†
187

$(11.1 \%)$
1

aPR $[95 \%$

Cl]‡ p

value
0.373

326

(10.2\%)

0.013

\section{4}

(12.5\%)

$0.92[0.77$, 1.10]
$1.12[0.75$, 1.68]

${ }^{1}$ GISD German Index of Socioeconomic Deprivation, ${ }^{2}$ MSM Men who have sex with men

TStrata specific number and proportion of infections at the stage of AIDS at the time of diagnosis.

\#Prevalence ratios with corresponding $95 \%$ confidence intervals of infections at the stage of AIDS at the time of diagnosis were calculated for the exposure variable GISD using multivariable Poisson regression with ZIP code cluster-robust error variance (standard errors were adjusted for 644 clusters). The model was stratified for MSM and adjusted for the variables of approximated age at the time of infection, region of origin and city size. For simplicity purposes, only strata specific effect estimates of the GISD conditional on countryside vs. town/major city are depicted. The effect estimates of the remaining covariates are nearly identical as presented in Table 4 in the MSM stratum.

Plasma Anti-CD4 Autoreactive IgG and Its Association With CD4+ T-Cell Recovery in Acute HIVInfected Individuals. Front Pharmacol. 2020;11(449).

2. HIV Treatment. Centers for Disease Control and Prevention. 2020.

https://www.cdc.gov/hiv/basics/livingwithhiv/treatment.html. Accessed 13 Nov 2020.

3. Sharma S, Schlusser KE, de la Torre P, Tambussi G, Draenert R, Pinto AN, et al. The benefit of immediate compared with deferred antiretroviral therapy on CD4+ cell count recovery in early HIV infection AIDS. 2019;33(8):1335-44.

4. Fleishman J, Yehia B, Moore R, Gebo K, Network HR. The economic burden of late entry into medical care for patients with HIV infection. Med Care. 2010;48(12):1071-9. 
5. Halperin J, Katz M, Pathmanathan I, Myers L, Van Sickels N, Seal P, et al. Early HIV Diagnosis Leads to Significantly Decreased Costs in the First 2 Years of HIV Care in an Urban Charity Hospital in New Orleans. J Int Assoc Provid AIDS Care.16(6):527-30.

6. Li Z, Purcell SL, Sansom SL, Hayes D, Hall HI. Vital Signs: HIV Transmission Along the Continuum of Care - United States, 2016. US Department of Health and Human Services/Centers for Disease Control and Prevention; 2019.

7. Rodger AJ, Cambiano V, Bruun T, Vernazza P, Collins S, Degen O, et al. Risk of HIV transmission through condomless sex in serodifferent gay couples with the HIV-positive partner taking suppressive antiretroviral therapy (PARTNER): final results of a multicentre, prospective, observational study. Lancet. 2019;393(10189):2428-38.

8. UNAIDS. Fast-Track - Ending the AIDS epidemic by 2030. 2014. https://www.unaids.org/sites/default/files/media_asset/JC2686_WAD2014report_en.pdf.

9. Accessed 16 Nov 2020.

10. Consolidated guidelines on HIV testing services for a changing epidemic. World Health Organization; 2019. Report No.: WHO/CDS/HIV/19.31.

11. HIV/AIDS surveillance in Europe 2019. 2018 data. Stockholm: European Centre for Disease Prevention and Control/WHO Regional Office for Europe; 2019.

12. Tavoschi L, Gomes Dias J, Pharris A. New HIV diagnoses among adults aged 50 years or older in 31 European countries, 2004-15: an analysis of surveillance data. Lancet HIV. 2017;4(11):514-21.

13. HIV im Jahr 2019 in Deutschland: Neuinfektionen und Gesamtkrankheitslast. Epidemiologisches Bulletin: Robert Koch-Institut; 2020. Report No.: 48.

14. Hofmann A, Hauser A, Zimmermann R, Santos-Hövener C, Bätzing-Feigenbaum J, Wildner S, et al. Surveillance of recent HIV infections among newly diagnosed HIV cases in Germany between 2008 and 2014. BMC Infect Dis. 2017;17(484).

15. Glymour MM, Avendano M, Kawachi I. Socioeconomic Status and Health. Social Epidemiology. 2 ed: Oxford University Press; 2014.

16. del Amo J, Lodi S, Dray-Spira R, Wittkop L, Monge S, Braun D, et al. Inequalities by educational level in response to combination antiretroviral treatment and survival in HIV-positive men and women in Europe. AIDS. 2017;31(2):253-62.

17. Pellowski JA, Kalichman SC, Matthews KA, Adler N. A pandemic of the poor: social disadvantage and the U.S. HIV epidemic. Am Psychol. 2013;68(4):197-209.

18. Rubin MS, Colen CG, Link BG. Examination of inequalities in HIV/AIDS mortality in the United States from a fundamental cause perspective. Am J Public Health. 2010;100(6):1053-9.

19. Kroll LE, Schumann M, Hoebel J, Lampert T. Regionale Unterschiede in der Gesundheit - Entwicklung eines sozioökonomischen Deprivationsindex für Deutschland. J Health Monit. 2017;2(2):103-20.

20. Calypte. Aware ${ }^{\text {TMMED }}{ }^{\text {TM}}$ EIA HIV-1 Incidence Test (IgG-Capture HIV-EIA). Enzyme Immunoassay for Population Esitmates of HIV-1 Incidence. Portland, USA: Calypte Biomedical Corporation; 2008. 
21. Corporation SB. Sedia ${ }^{\mathrm{T}}$ BED HIV-1 Incidence EIA. Enzyme Immunoassay for Population Esitmates of HIV-1 Incidence. Portland, USA: Sedia Biosciences Corporation; 2014.

22. Loschen S, Bätzing-Feigenbaum J, Poggensee G, Cordes C, Hintsche B, Rausch M, et al. Comparison of the human immunodeficiency virus (HIV) type 1-specific immunoglobulin $\mathrm{G}$ capture enzyme-linked immunosorbent assay and the avidity index method for identification of recent HIV infections. J Clin Microbiol. 2008;46(1):341-5.

23. Fortschreibung des Bevölkerungsstandes. Statistisches Bundesamt (Destatis). 2019. https://wwwgenesis.destatis.de/genesis/online?

operation=statistic $\&$ levelindex $=0 \&$ levelid $=1634473871845 \&$ code $=12411 \#$ abreadcrumb. Accessed 24 Mar 2020.

24. 1993 Revised Classification System for HIV Infection and Expanded Surveillance Case Definition for AIDS Among Adolescents and Adults Centers for Disease Control and Prevention; 1992

25. Kroll LE, Schumann M, Hoebel J, Lampert T. German Index of Socioeconomic Deprivation (GISD) Version 1.0. GESIS Datenarchiv 2017. http://doi.org/10.7802/1460.

26. Collaborative Group on AIDS Incubation and HIV Survival including the CASCADE EU Concerted Action. Time from HIV-1 seroconversion to AIDS and death before widespread use of highly-active antiretroviral therapy: a collaborative re-analysis. Lancet. 2000;355(9210):1131-7.

27. Giesecke J, Scalia-Tomba G, Håkansson C, Karlsson A, Lidman K. Incubation time of AIDS: progression of disease in a cohort of HIV-infected homo- and bisexual men with known dates of infection. Scand J Infect Dis. 1990;22(4):407-11.

28. Mau M. Das Landei: HIV-Versorgung außerhalb der Großstädte. Esanum. 2018. https://www.esanum.de/fachbereichsseite-dermatologie/feeds/dermatologie/conferences/AIDSHepatitis-18/feeds/today/posts/das-landei-hiv-versorgung-auerhalb-der-grostadte. Accessed 23 Nov 2020.

29. Zoufaly A, an der Heiden M, Marcus U, Hoffmann C, Stellbrink H, Voss L, et al. Late presentation for HIV diagnosis and care in Germany. HIV Med. 2012;13(3):172-81.

30. Noble G, Okpo E, Tonna I, Fielding S. Factors associated with late HIV diagnosis in North-East Scotland: a six-year retrospective study. Public Health. 2016;139:36-43.

31. Smith RD, Delpech VC, Brown AE, Rice BD. HIV transmission and high rates of late diagnoses among adults aged 50 years and over. AIDS. 2010;24(13):2109-15.

32. Wohlgemut J, Lawes T, Laing RB. Trends in missed presentations and late HIV diagnosis in a UK teaching hospital: a retrospective comparative cohort study. BMC Infec Dis. 2012;12(72).

33. Buot MLG, Docena JP, Ratemo BK, Bittner MJ, Burlew JT, Nuritdinov AR, et al. Beyond race and place: Distal sociological determinants of HIV disparities. PLoS ONE. 2014;9(4).

34. Denning P, DiNenno E. Communities in Crisis: Is There a Generalized HIV Epidemic in Impoverished Urban Areas of the United States? [Abstract]. XVIII International AIDS Conference, Vienna, Austria 18-23 July 2010. 
35. Ransome Y, Kawachi I, Braunstein S, Nash D. Structural inequalities drive late HIV diagnosis: The role of black racial concentration, income inequality, socioeconomic deprivation, and HIV testing. Health Place. 2016;42:148-58.

36. Matl M. HIV: Späte Diagnose bei Heterosexuellen. Österreichischer Rundfunk. 2019. https://noe.orf.at/stories/3023018/. Accessed 14 Dec 2020.

37. Langer PC. Spätdiagnose HIV: Hintergründe und Verbesserungsansätze für die Präventionsarbeit Deutschen AIDS-Hilfe e.V.; 2010.

38. Lodi S, Dray-Spira R, Touloumi G, Braun D, Teira R, D'Arminio Monforte A, et al. Delayed HIV diagnosis and initiation of antiretroviral therapy: inequalities by educational level, COHERE in EuroCoord. AIDS. 2014;24(15):2297-306.

39. Walcott M, Kempf MC, Merlin JS, Turan JM. Structural community factors and sub-optimal engagement in HIV care among low-income women in the Deep South of the USA. Cult Health Sex. 2016;18(6):682-94.

40. Hendrich T. Ausgeprägte Gay Szene - Wo leben die meisten Schwulen? Netzsieger. 2018. https://www.netzsieger.de/ratgeber/wo-leben-die-meisten-schwulen. Accessed 14 Dec 2020.

41. Schink S, Schafberger A, Tappe M, Marcus U. Gemeinsames Teststellenprojekt 2017. Robert KochInstitut. 2019; doi:10.25646/5847.3

42. Hoebel J, Kroll LE, Fiebig J, Lampert T, Katalinic A, Barnes B, et al. Socioeconomic Inequalities in Total and Site-Specific Cancer Incidence in Germany: A Population-Based Registry Study. Front Oncol. 2018;8(402).

\section{Discussion}

This study investigated the impact of regional socioeconomic deprivation on late HIV diagnoses in Germany. The proportion of long-standing infections as well as AIDS at the time of diagnosis was somewhat higher in regions of high socioeconomic deprivation compared to regions with low and medium deprivation. The results of the stratified multivariable analyses showed that MSM living in highly deprived regions in the countryside were more likely to have a long-standing infection and also more likely to have already developed an AIDS-defining condition at the time of their diagnosis compared to MSM in less deprived countryside regions. This effect of deprivation was not observed among MSM who live in towns or major cities. Among persons who acquired HIV via heterosexual contact, no effect of regional socioeconomic deprivation on the timing of their diagnosis was observed. Since the proportion of late diagnoses was substantially higher in the heterosexual transmission group compared to MSM, the results suggest that persons with heterosexual contact are generally at a higher risk of presenting late irrespective of their socioeconomic background. The proportion of late diagnoses among MSM was lower, for this transmission group socioeconomic deprivation in regions in the countryside however appears to have an impact on the timing of HIV diagnosis. 
Previous studies in the German context have mainly focused on the distribution of late HIV presentation by available sociodemographic data of the national case surveillance [28]. The findings of our study are consistent with these as the highest proportions of late diagnoses were also observed among persons with heterosexual contact, older age at the time of diagnosis and migrants from high-prevalence countries $[12,13,28]$. These patterns have remained stable over time and were also observed in other Western European and North American countries [11, 29-31]. The relationship between limited socioeconomic resources and HIV has found attention especially in research conducted in the US where several studies found that the prevalence of advanced HIV infections was higher in impoverished areas and that structural inequalities drove late diagnoses [17, 32-34].

The findings of our study therefore build on existing evidence with regard to the population groups who are more likely to be diagnosed at later stages of their HIV infection. It is assumed that persons with heterosexual contact often do not test for HIV due to a lower self-perception of risk as well as limited knowledge and stigma-related misconceptions about HIV [35]. At the same time, this low awareness of HIV in the heterosexual transmission group is also existent among health care providers, who as a result often do not consider an HIV infection even if indicator illnesses are present [36]. As MSM have been and still are the population group most affected by HIV in the Western context, health campaigns promoting early testing behaviours have predominantly targeted this group [13]. With respect to socioeconomic deprivation, this study's results also confirm the findings of previous research in other Western contexts, showing that late diagnoses are more prevalent in areas of high socioeconomic deprivation. This has been attributed to the fact that people with fewer socioeconomic resources generally have lower health literacy, are less likely to practice healthy behaviours and have worse access to treatment $[33,37,38]$.

Our study's findings however provide new insights into the differences between the main transmission groups as well as the role of city size, seeing that after controlling for the confounding variables, an effect of socioeconomic deprivation was found in MSM from countryside areas but not in MSM from towns or bigger cities, and on the whole not in persons with heterosexual contact. A possible hypothesis might be that socioeconomic factors that are usually associated with health outcomes do not play a role among persons with heterosexual contact because the risk perception of HIV in this group is generally low and outweighs the expected impact of socioeconomic inequalities. HIV education traditionally had a stronger focus on MSM, which might explain why MSM on the other hand are affected by a lack of socioeconomic resources when it comes to HIV health outcomes. As the assessment of MSM who live in countryside versus urban areas showed that the observed effect among MSM was driven by persons from the countryside, it can be assumed that urban areas offer better support structures for this group. The fact that HIV checkpoints, specialised medical practices and MSM communities are more often located in towns and bigger cities $[27,39,40]$ might help to offset the effect of socioeconomic deprivation for MSM in urban areas when it comes to the detection of HIV. These findings should be taken into consideration when developing strategies aimed at reducing the number of late HIV diagnoses in Germany. The results suggest that it is necessary to target persons with heterosexual contact irrespective of their socioeconomic background. Structural inequalities can be considered when MSM are addressed, with an increased focus on rural regions of higher socioeconomic deprivation for this population group. 
A strength of this study is the utilisation of the GISD in the analysis of the impact of regional socioeconomic deprivation. As the score was calculated for each region nationwide, it was possible to match a score to every patient included in the analysis on the basis of the ZIP code, allowing to analyse the majority of all newly diagnosed HIV cases reported between 2011 and 2018. This comprehensiveness might not have been ensured if socioeconomic status had been indicated by the patients or respective doctors. In addition to that, the GISD has shown to be a reliable tool for the analysis of regional socioeconomic inequalities and health as statistical links were also found in prior research concerning life expectancy, major causes of death and various behavioural health risks in Germany $[18,41]$.

This study is also subject to limitations. Both outcome variables had missing values (see Table $\mathrm{S} 1$ and Table S2, Additional file 2) due to the fact that the BED-CEIA is not performed for all newly diagnosed cases and the CDC category not indicated in some cases. However, as the variables were reported by laboratories and physicians, it is presumed that there was no relationship between the probability of an outcome value being missing and the outcome itself, and hence no missingness pattern discernible that might have considerably affected the analysis. Another limitation is the fact that the GISD illustrates regional and not individual socioeconomic deprivation. It is therefore beyond the scope of this study to make claims about the impact of a person's individual socioeconomic status on late HIV presentation. Nevertheless, as risk factors regarding health outcomes are generally more prevalent in areas characterised by high levels of socioeconomic deprivation, regional socioeconomic factors have proven to serve as valid proxy variables for aggregated individual socioeconomic deprivation [18].

This study offers new findings and insights into the relationship of regional socioeconomic deprivation and late HIV presentation in the context of Germany. Further research that builds on these findings is therefore needed. We recommend that future studies also take individual socioeconomic factors into account. In order to gain a deeper understanding of the underlying dynamics, it would also be of interest to investigate which HIV health behaviours as well as structural factors related to the healthcare system are most affected by socioeconomic deprivation and how these could be best addressed.

\section{Conclusions}

This study has found that HIV infections which are diagnosed at long-standing and AIDS stages are somewhat more prevalent in areas characterised by high levels of socioeconomic deprivation. After having controlled for confounding variables, an effect of socioeconomic deprivation on the timing of HIV diagnosis was however found only among MSM from countryside areas. In order to tackle the burden of late HIV diagnoses in Germany, the findings suggest that efforts in promoting HIV awareness and regular testing behaviours have to be increased for heterosexual persons irrespective of their socioeconomic background. For MSM, we recommend that a focus is put on those living in rural regions of higher socioeconomic deprivation.

\section{Declarations}




\section{Ethics approval and consent to participate}

The "InzSurv-HIV" study has been approved by the data protection officer of the Robert Koch Institute and the Federal Commissioner for Data Protection and Freedom of Information (II-401/008\#0016). Ethical approval for the monitoring of recent HIV infections was given by the ethics commission at the Charité University Medicine Berlin (EA1/007/08). No patient informed consent was obtained as the data on new HIV diagnoses were derived from the national statutory HIV surveillance which is carried out in compliance with the German Infection Protection Act (IfSG). The samples used in the BED-CEIA assay were residuals from routine diagnostic processing. Recency testing of these samples is only licensed for epidemiological analyses and does not allow for individual patient analyses. All methods used in our study were carried out in accordance with relevant guidelines and regulations.

\section{Consent for publication}

Not applicable.

\section{Availability of data and materials}

The HIV data analysed during the current study are not publicly available. They were generated from the national statutory notification of newly diagnosed HIV cases and blood samples which are directly sent and processed at the Robert Koch Institute. The dataset is available from the corresponding author on reasonable request.

The German Index of Socioeconomic Deprivation is publicly available and can be accessed via the GESIS repository, http://doi.org/10.7802/1460.

\section{Competing interests}

The authors declare that they have no competing interests.

\section{Funding}

This study was supported by the corresponding author's doctoral grant from the Robert Koch Institute.

\section{Authors' contributions}

AP, UK, BGB and VB designed the study. NB and KH were in charge of the BED-CEIA laboratory tests and provided the data. JH and NM conceived the GISD and provided the data as well as guidance on its use in health research. AP and UK coordinated the study and performed the analyses. MadH provided input for the statistical analyses. AP drafted the manuscript and all authors contributed to reviewing and revising the draft.

\section{Acknowledgements}


We would like to thank all participating laboratories for sending dried filter spots for laboratory analyses. Laboratories that have agreed to publish their names are listed at https://www.rki.de/DE/Content/InfAZ/H/HIVAIDS/Studien/InzSurv_HIV/beteiligte_Labore.html. We also thank Dr. Andrea Hauser for her contribution to the recency testing, the RKI staff assisting in the laboratory tests as well as the staff documenting the test results and data from the statutory notification of newly diagnosed HIV cases. We would also like to thank Prof. Tobias Kurth and Jessica Rohmann (both Institute of Public Health, Charité - University Medicine Berlin) for their guidance in the conception and usage of DAGs in public health research.

\section{References}

1. Song A, Li Z, Luo Z, Lu X, Wang R, Liu L, et al. Effects of Early and Delayed Antiretroviral Therapy on Plasma Anti-CD4 Autoreactive IgG and Its Association With CD4+ T-Cell Recovery in Acute HIV-Infected Individuals. Front Pharmacol. 2020;11(449).

2. HIV Treatment. Centers for Disease Control and Prevention. 2020.

https://www.cdc.gov/hiv/basics/livingwithhiv/treatment.html. Accessed 13 Nov 2020.

3. Sharma S, Schlusser KE, de la Torre P, Tambussi G, Draenert R, Pinto AN, et al. The benefit of immediate compared with deferred antiretroviral therapy on CD4+ cell count recovery in early HIV infection AIDS. 2019;33(8):1335-44.

4. Fleishman J, Yehia B, Moore R, Gebo K, Network HR. The economic burden of late entry into medical care for patients with HIV infection. Med Care. 2010;48(12):1071-9.

5. Halperin J, Katz M, Pathmanathan I, Myers L, Van Sickels N, Seal P, et al. Early HIV Diagnosis Leads to Significantly Decreased Costs in the First 2 Years of HIV Care in an Urban Charity Hospital in New Orleans. J Int Assoc Provid AIDS Care.16(6):527-30.

6. Li Z, Purcell SL, Sansom SL, Hayes D, Hall HI. Vital Signs: HIV Transmission Along the Continuum of Care - United States, 2016. US Department of Health and Human Services/Centers for Disease Control and Prevention; 2019.

7. Rodger AJ, Cambiano V, Bruun T, Vernazza P, Collins S, Degen O, et al. Risk of HIV transmission through condomless sex in serodifferent gay couples with the HIV-positive partner taking suppressive antiretroviral therapy (PARTNER): final results of a multicentre, prospective, observational study. Lancet. 2019;393(10189):2428-38.

8. UNAIDS. Fast-Track - Ending the AIDS epidemic by 2030. 2014.

https://www.unaids.org/sites/default/files/media_asset/JC2686_WAD2014report_en.pdf.

Accessed 16 Nov 2020.

Page 22/25 
9. Consolidated guidelines on HIV testing services for a changing epidemic. World Health Organization; 2019. Report No.: WHO/CDS/HIV/19.31.

10. HIV/AIDS surveillance in Europe 2019. 2018 data. Stockholm: European Centre for Disease Prevention and Control/WHO Regional Office for Europe; 2019.

11. Tavoschi L, Gomes Dias J, Pharris A. New HIV diagnoses among adults aged 50 years or older in 31 European countries, 2004-15: an analysis of surveillance data.Lancet HIV. 2017;4(11):514-21.

12. HIV im Jahr 2019 in Deutschland: Neuinfektionen und Gesamtkrankheitslast. Epidemiologisches Bulletin: Robert Koch-Institut; 2020. Report No.: 48.

13. Hofmann A, Hauser A, Zimmermann R, Santos-Hövener C, Bätzing-Feigenbaum J, Wildner S, et al.Surveillance of recent HIV infections among newly diagnosed HIV cases in Germany between 2008 and 2014. BMC Infect Dis. 2017;17(484).

14. Glymour MM, Avendano M, Kawachi I. Socioeconomic Status and Health. Social Epidemiology. 2 ed: Oxford University Press; 2014.

15. del Amo J, Lodi S, Dray-Spira R, Wittkop L, Monge S, Braun D, et al. Inequalities by educational level in response to combination antiretroviral treatment and survival in HIV-positive men and women in Europe. AIDS. 2017;31(2):253-62.

16. Pellowski JA, Kalichman SC, Matthews KA, Adler N. A pandemic of the poor: social disadvantage and the U.S. HIV epidemic. Am Psychol. 2013;68(4):197-209.

17. Rubin MS, Colen CG, Link BG. Examination of inequalities in HIV/AIDS mortality in the United States from a fundamental cause perspective.Am J Public Health. 2010;100(6):1053-9.

18. Kroll LE, Schumann M, Hoebel J, Lampert T. Regionale Unterschiede in der Gesundheit - Entwicklung eines sozioökonomischen Deprivationsindex für Deutschland.J Health Monit. 2017;2(2):103-20.

19. Calypte. Aware ${ }^{\text {TM }} B E D^{\text {TM }} E I A$ HIV-1 Incidence Test (IgG-Capture HIV-EIA). Enzyme Immunoassay for Population Esitmates of HIV-1 Incidence. Portland, USA: Calypte Biomedical Corporation; 2008.

20. Corporation SB. Sedia ${ }^{\mathrm{T}}$ BED HIV-1 Incidence EIA. Enzyme Immunoassay for Population Esitmates of HIV-1 Incidence. Portland, USA: Sedia Biosciences Corporation; 2014.

21. Loschen S, Bätzing-Feigenbaum J, Poggensee G, Cordes C, Hintsche B, Rausch M, et al. Comparison of the human immunodeficiency virus (HIV) type 1-specific immunoglobulin G capture enzyme-linked immunosorbent assay and the avidity index method for identification of recent HIV infections.J Clin Microbiol. 2008;46(1):341-5. 
22. Fortschreibung des Bevölkerungsstandes. Statistisches Bundesamt (Destatis). 2019. https://wwwgenesis.destatis.de/genesis/online?

operation=statistic\&levelindex $=0 \&$ levelid $=1634473871845 \&$ code $=12411$ \#abreadcrumb.Accessed 24 Mar 2020 .

23. 1993 Revised Classification System for HIV Infection and Expanded Surveillance Case Definition for AIDS Among Adolescents and Adults Centers for Disease Control and Prevention; 1992

24. Kroll LE, Schumann M, Hoebel J, Lampert T. German Index of Socioeconomic Deprivation (GISD) Version 1.0. GESIS Datenarchiv 2017. http://doi.org/10.7802/1460.

25. Collaborative Group on AIDS Incubation and HIV Survival including the CASCADE EU Concerted Action. Time from HIV-1 seroconversion to AIDS and death before widespread use of highly-active antiretroviral therapy: a collaborative re-analysis. Lancet. 2000;355(9210):1131-7.

26. Giesecke J, Scalia-Tomba G, Håkansson C, Karlsson A, Lidman K. Incubation time of AIDS: progression of disease in a cohort of HIV-infected homo- and bisexual men with known dates of infection.Scand J Infect Dis. 1990;22(4):407-11.

27. Mau M. Das Landei: HIV-Versorgung außerhalb der Großstädte. Esanum. 2018. https://www.esanum.de/fachbereichsseite-dermatologie/feeds/dermatologie/conferences/AIDSHepatitis-18/feeds/today/posts/das-landei-hiv-versorgung-auerhalb-der-grostadte.Accessed 23 Nov 2020.

28. Zoufaly A, an der Heiden M, Marcus U, Hoffmann C, Stellbrink H, Voss L, et al.Late presentation for HIV diagnosis and care in Germany. HIV Med. 2012;13(3):172-81.

29. Noble G, Okpo E, Tonna I, Fielding S. Factors associated with late HIV diagnosis in North-East Scotland: a six-year retrospective study. Public Health. 2016;139:36-43.

30. Smith RD, Delpech VC, Brown AE, Rice BD. HIV transmission and high rates of late diagnoses among adults aged 50 years and over. AIDS. 2010;24(13):2109-15.

31. Wohlgemut J, Lawes T, Laing RB. Trends in missed presentations and late HIV diagnosis in a UK teaching hospital: a retrospective comparative cohort study. BMC Infec Dis. 2012;12(72).

32. Buot MLG, Docena JP, Ratemo BK, Bittner MJ, Burlew JT, Nuritdinov AR, et al. Beyond race and place: Distal sociological determinants of HIV disparities. PLoS ONE. 2014;9(4).

33. Denning P, DiNenno E. Communities in Crisis: Is There a Generalized HIV Epidemic in Impoverished Urban Areas of the United States? [Abstract]. XVIII International AIDS Conference, Vienna, Austria - 18-23 July 2010. 
34. Ransome Y, Kawachi I, Braunstein S, Nash D. Structural inequalities drive late HIV diagnosis: The role of black racial concentration, income inequality, socioeconomic deprivation, and HIV testing. Health Place. 2016;42:148-58.

35. Matl M. HIV: Späte Diagnose bei Heterosexuellen. aÖsterreichischer Rundfunk. 2019. https://noe.orf.at/stories/3023018/. Accessed 14 Dec 2020.

36. Langer PC. Spätdiagnose HIV: Hintergründe und Verbesserungsansätze für die Präventionsarbeit Deutschen AIDS-Hilfe e.V.; 2010.

37. Lodi S, Dray-Spira R, Touloumi G, Braun D, Teira R, D'Arminio Monforte A, et al. Delayed HIV diagnosis and initiation of antiretroviral therapy: inequalities by educational level, COHERE in EuroCoord. AIDS. 2014;24(15):2297-306.

38. Walcott M, Kempf MC, Merlin JS, Turan JM. Structural community factors and sub-optimal engagement in HIV care among low-income women in the Deep South of the USA.Cult Health Sex. 2016;18(6):682-94.

39. Hendrich T. Ausgeprägte Gay Szene - Wo leben die meisten Schwulen? Netzsieger. 2018. https://www.netzsieger.de/ratgeber/wo-leben-die-meisten-schwulen. Accessed 14 Dec 2020.

40. Schink S, Schafberger A, Tappe M, Marcus U. Gemeinsames Teststellenprojekt 2017. Robert KochInstitut.2019; doi:10.25646/5847.3

41. Hoebel J, Kroll LE, Fiebig J, Lampert T, Katalinic A, Barnes B, et al. Socioeconomic Inequalities in Total and Site-Specific Cancer Incidence in Germany: A Population-Based Registry Study. Front Oncol. 2018;8(402).

\section{Supplementary Files}

This is a list of supplementary files associated with this preprint. Click to download.

- Additionalfile1DAG.docx

- Additionalfile2Missingvalues.docx 